\title{
Análisis De Los Niveles De Logro De Aprendizaje Matemático En El Sistema Escolar Ecuatoriano
}

\author{
Janneth Morocho, Máster, \\ Carlos Buenaño, Máster, \\ Martha Dávalos, Matemática \\ Escuela Superior Politécnica de Chimborazo / Ecuador
}

Doi:10.19044/esj.2020.v16n24p199 URL:http://dx.doi.org/10.19044/esj.2020.v16n24p199

\section{Resumen}

El presente artículo refleja el trabajo de un grupo de investigación que pretende investigar los niveles de logro de aprendizaje en matemática desarrollados por los estudiantes del sistema escolar ecuatoriano. Se ha considerado como fuente de información al Instituto Nacional de Evaluación Educativa (INEVAL) de la República de Ecuador, quienes publican periódicamente los resultados de las evaluaciones realizadas a los estudiantes de cuarto, séptimo y décimo de educación básica, así como a los estudiantes de tercer año de bachillerato. Se realizó un análisis descriptivo y una prueba ANOVA de los datos de los periodos escolares de estudio. Al igual que en el resto de la región los resultados se replican en Ecuador, mostrando que un alto porcentaje de estudiantes del sistema escolar ecuatoriano tienen entre nivel insuficiente y elemental.

Palabras clave: Logro de aprendizaje, Matemática 


\title{
Analysis Of Levels Of Achievement Of Mathematical Learning In The Ecuadorian School System
}

\author{
Janneth Morocho, Máster, \\ Carlos Buenaño, Máster, \\ Martha Dávalos, Matemática
}

Escuela Superior Politécnica de Chimborazo / Ecuador

\begin{abstract}
This paper focuses on investigating the levels of learning achievement in mathematics, developed by students of the Ecuadorian school system. The National Institute of Educational Evaluation (NIEE) of the Republic of Ecuador has been considered as a source of information. This institute periodically publishes the results of the evaluations made to the fourth, seventh and tenth students of basic education, as well as to third year students. A descriptive analysis and an ANOVA test of the data of the school periods of study were performed. In the rest of the region, the results are replicated in Ecuador. This, however, shows that a high percentage of students in the Ecuadorian school system had insufficient education and were at elementary level.
\end{abstract}

Keywords: Learning achievement, Mathematics

\section{Introducción}

De acuerdo al objetivo planteado, se utilizan para el análisis dos aspectos: uno referido a los niveles de logro de aprendizaje en la asignatura de matemática y otro referido a los estudiantes del sistema escolar ecuatoriano.

En cuanto a los niveles de logro de aprendizaje en matemática, se considerarán en primer lugar, los aportes de Ortiz (2005) quien define los logros de aprendizaje como "un modelo pedagógico del encargo social que refleja los propósitos, metas y aspiraciones a alcanzar por el estudiante, desde el punto de vista cognitivo e instrumental”. En segundo lugar, se tomará en cuenta también el aprendizaje definido por David Ausubel (aprendizaje significativo): "el conocimiento verdadero solo puede nacer cuando los nuevos contenidos tienen un significado a la luz de los conocimientos que ya se tienen" (Ausubel, 1983). Finalmente, se entenderá también como niveles de logro los definidos por el Instituto Nacional de Evaluación Educativa (INEVAL), institución gubernamental, responsable de la evaluación integral, 
externa e interna, del Sistema Nacional de Educación del Ecuador (Instituto Nacional de Evaluación Educativa, 2012).

El INEVAL aplica anualmente y de manera muestral las evaluaciones Ser Estudiante a los estudiantes de cuarto, séptimo y décimo año de Educación General Básica, mientras que a tercer año de Bachillerato aplica la prueba Ser Bachiller. La evaluación cuenta con instrumentos que permiten determinar el nivel de logro de los estudiantes con respecto a los estándares de aprendizaje establecidos por el Ministerio de Educación (MINEDUC). También incluyen encuestas de Factores Asociados cuyo objetivo es contextualizar las características del medio en el que se desenvuelven los estudiantes y describir cómo estos factores inciden en sus logros académicos (INEVAL, 2016).

Las áreas de interés que se evalúan son: Matemática, Lengua y Literatura, Ciencias Naturales y Estudios Sociales. La nota de las evaluaciones se asigna mediante un puntaje que va de 0 a 1000 puntos, ubicando a cada estudiante en un nivel de logro acorde a su puntaje y su grado de dominio de los estándares (ver Tabla 1).

Tabla 1. Equivalencias entre puntaje y nivel de logro

\begin{tabular}{|c|c|}
\hline PUNTAJE & $\begin{array}{c}\text { NIVEL DE } \\
\text { LOGRO }\end{array}$ \\
\hline $0-699$ & Insuficiente \\
\hline $700-799$ & Elemental \\
\hline $800-949$ & Satisfactorio \\
\hline $950-1000$ & Excelente \\
\hline
\end{tabular}

Las evaluaciones tienen carácter muestral y se realizan cada ciclo escolar. Cada muestra es estratificada por área rural-urbana, tipo de sostenimiento (fiscales, fiscomisionales, privadas y municipales) y régimen escolar (sierra, costa), además se consideran las proporciones de sexo femenino y masculino con un nivel de confianza del $95 \%$.

Estas evaluaciones demuestran que existe un alto porcentaje de estudiantes cuyos niveles de logro de aprendizaje en matemática se encuentran entre insuficiente y elemental.

\section{Texto principal}

Algunos de los logros de aprendizaje en matemática definidos por el MINEDUC para los estudiantes de cuarto, séptimo y décimo de educación básica así como para los estudiantes de tercero de bachillerato se describen a continuación (Ministerio de Educación, 2016):

\section{Cuarto año de básica}

- Relaciona las operaciones aritméticas con la construcción de patrones de figuras y numéricos, para desarrollar el pensamiento lógico matemático.

- Resuelve situaciones reales de la cotidianidad que están relacionadas con la estimación, medición, uso de unidades convencionales y no convencionales, 
operaciones aritméticas y refuerza valores como la honestidad, respeto e integridad en su diario vivir.

- Utiliza la información del entorno para sacar conclusiones mediante el uso de la estadística descriptiva, desarrollando así el pensamiento lógico matemático, participando en proyectos de análisis de datos.

\section{Séptimo año de básica}

- Utiliza las operaciones aritméticas para solucionar problemas del entorno, desarrollando el pensamiento lógico matemático y comprendiendo los modelos matemáticos.

- Desarrolla algoritmos de las operaciones aritméticas con números naturales, decimales y fracciones para solucionar problemas de la cotidianidad.

- Resuelve problemas que requieren el desarrollo de cálculos de perímetros y áreas de polígonos regulares; la estimación y medición de longitudes, áreas, volúmenes y masas de objetos; la conversión de unidades y el uso de la tecnología, para comprender el espacio donde se desenvuelve.

- Utiliza las TIC para representar la información estadística y calcula e interpreta las medidas de tendencia central.

\section{Décimo año de básica}

- Resuelve operaciones aritméticas con números reales mediante la comprensión de sus propiedades, desarrollando el pensamiento lógico creativo.

- Resuelve problemas reales mediante la aplicación del teorema de Pitágoras y las fórmulas para el cálculo de perímetros, áreas, volúmenes, ángulos de cuerpos y figuras geométricas.

- Resuelve problemas que involucran la conversión de unidades de medida del SI y de otros sistemas, el perímetro y área de figuras planas, áreas y volúmenes de cuerpos geométricos.

- Desarrolla el pensamiento crítico mediante la representación, análisis e interpretación de datos estadísticos y situaciones relacionadas con la probabilidad y el uso de las TIC, para conocer y comprender mejor el entorno social y económico.

\section{Tercer año de bachillerato}

- Propone soluciones creativas de problemas concretos de la realidad nacional y mundial mediante el uso de las operaciones básicas de los diferentes conjuntos numéricos y de funciones reales de variable real, algoritmos, estrategias y métodos de razonamiento matemático, que permiten juzgar con responsabilidad la validez de procedimientos y los resultados en un contexto. 
- Interpreta y soluciona problemas del medio, desarrollando estrategias individuales y grupales que contribuyen al cálculo mental y escrito, exacto o estimado.

-Emplea las TIC para realizar cálculos y resolver problemas de la realidad nacional, argumentando la pertinencia de los métodos utilizados y juzgando la validez de los resultados.

-Vincula los conocimientos matemáticos con otras disciplinas científicas y saberes ancestrales para plantear soluciones de problemas reales.

Algunos ejemplos de preguntas de la evaluación Ser Estudiante para estudiantes de cuarto año se observan en la ilustración 1.
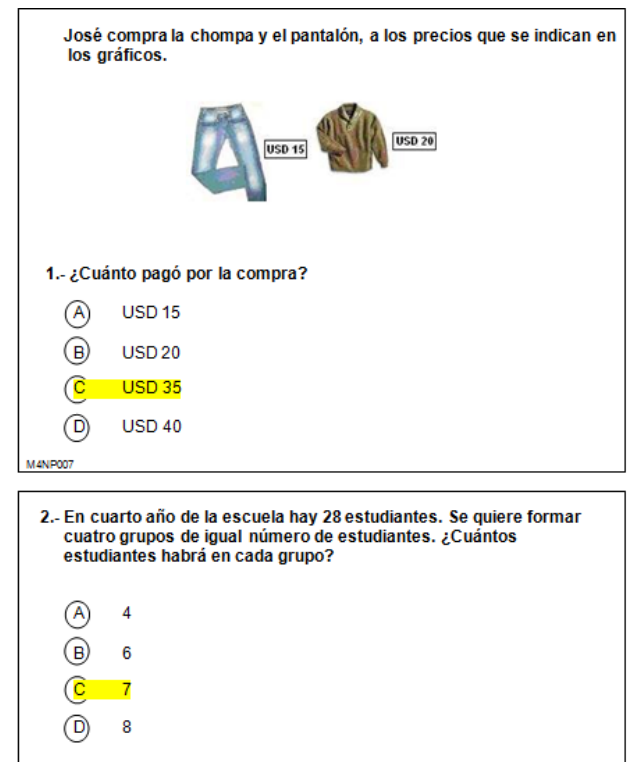

Ilustración 1. Ejemplo de preguntas de la evaluación Ser Estudiante. Cuarto año de básica.

A partir del año 2013 el INEVAL publica los resultados de las evaluaciones realizadas en todo el Ecuador a estudiantes de cuarto, séptimo y décimo de educación básica, así como los resultados de tercero de bachillerato en las asignaturas: Matemática, Lengua y Literatura, Estudios Sociales y Ciencias Naturales.

Los resultados publicados por el INEVAL y analizados por los investigadores, corresponden a las evaluaciones de los ciclos escolares, 20132014, 2014- 2015 y 2015-2016 en los que se evaluaron a 25309, 25651 y 28904 estudiantes respectivamente.

Es importante remarcar que los padres de familia de los estudiantes de tercero de bachillerato contratan por fuera de las instituciones educativas, en centros de capacitación, programas que permitan rendir la prueba Ser Bachiller (Torres, 2016), pues como se indicó en párrafos anteriores esta 
prueba representa tanto un porcentaje de la nota de graduación, como el puntaje que le permitirá al estudiante ingresar a una carrera a nivel superior.

Metodología

La información analizada corresponde a las evaluaciones de dos pruebas, la prueba Ser Estudiante, dirigida a los niños y niñas de cuarto, séptimo y décimo de educación básica, y la prueba Ser Bachiller, dirigida a los estudiantes que finalizan el tercero de bachillerato. Para estos últimos esta prueba constituye el $30 \%$ de la nota de titulación. Dicha información corresponde a tres periodos: 2013-2014, 2014- 2015 y 2015-2016.

En primer lugar se aplicó la prueba estadística ANOVA, con un nivel de confianza de 0.95 e hipótesis nula H_0, mostrando que las medias de los porcentajes de los niveles de logro de aprendizaje en los diferentes periodos de estudio son iguales.

Luego de probar que no se puede rechazar la hipótesis nula; es decir, que el comportamiento de los estudiantes en lo que se refiere a los niveles de logro de aprendizaje durante los tres periodos de estudio es el mismo; se realizó un análisis descriptivo de los porcentajes de estudiantes que alcanzaron los diferentes niveles de logro de aprendizaje durante los tres años de estudio.

\section{Resultados}

- Con un nivel de confianza de 0.95 se probó que el comportamiento de los estudiantes en lo que se refiere a los niveles de logro de aprendizaje durante los tres periodos de estudio es el mismo.

- Se obtuvieron los siguientes resultados, que representan los niveles de logros de aprendizaje de matemática en promedio de los ciclos escolares 2013-2014, 2014- 2015 y 2015-2016.

Estos resultados están dados en porcentaje de acuerdo a la totalidad de estudiantes que rindieron las pruebas:

Tabla 2. Niveles de logros de aprendizaje en matemática de los estudiantes del sistema escolar ecuatoriano

\begin{tabular}{|c|c|c|c|c|}
\hline & $\begin{array}{c}\text { Insuficiente } \\
(\%)\end{array}$ & $\begin{array}{c}\text { Elemental } \\
(\%)\end{array}$ & $\begin{array}{c}\text { Satisfactorio } \\
(\%)\end{array}$ & $\begin{array}{c}\text { Excelente } \\
(\%)\end{array}$ \\
\hline Cuarto Año & 41 & 50,33 & 9 & 0 \\
\hline $\begin{array}{c}\text { Séptimo } \\
\text { Año }\end{array}$ & 37,67 & 45,67 & 17 & 0 \\
\hline $\begin{array}{c}\text { Décimo } \\
\text { Año }\end{array}$ & 41 & 50,33 & 9 & 0 \\
\hline $\begin{array}{c}\text { Tercero } \\
\text { Bachillerato }\end{array}$ & 28,67 & 51,67 & 20 & 0 \\
\hline Promedio & 37,08 & 49,5 & 13,75 & 0 \\
\hline
\end{tabular}

- Los estudiantes de tercero de bachillerato mejoran en un pequeño porcentaje el nivel de logro de aprendizaje debido a los refuerzos que 
estos reciben fuera de la institución educativa, más no por el esfuerzo realizado por los docentes dentro del aula. Algo similar ocurre con los estudiantes de séptimo de básica, pues en la mayoría de las instituciones educativas los estudiantes, además de la prueba Ser Estudiante, deben rendir una prueba de ingreso a octavo de básica.

\section{Conclusiones}

- El comportamiento en cuanto a los niveles de logro de aprendizaje matemático durante los periodos de evaluación es el mismo.

- El 37,08 \% de los estudiantes del sistema educativo ecuatoriano posee un nivel de logro de aprendizaje en matemática equivalente a insuficiente mientras que el 49,50 \% apenas alcanza el nivel de elemental; apenas un 13,17 \% posee un nivel de satisfactorio y no existen, en promedio, estudiantes con un nivel excelente.

\section{References:}

1. Ausubel, D.N. (1983). Psicología educativa. Un punto de vista cognoscitivo. México: Trillas.

2. INEVAL (2016). Resultados educativos, retos hacia la excelencia. Quito: INEVAL.

3. Instituto Nacional de Evaluación Educativa (2012). (26 de Noviembre de 2012). INSTITUTO NACIONAL DE EVALUACIÓN EDUCATIVA. Recuperado el 18 de Junio de 2019, de https://www.evaluacion.gob.ec/historia/

4. Ministerio de Educación (2016). (30 de Julio de 2016). www.educacion.gob.ec. Recuperado el, de chromeextension://oemmndcbldboiebfnladdacbdfmadadm/https://educacion. gob.ec/wpcontent/uploads/downloads/2016/03/MATE_COMPLETO.pdf

5. Ministerio de Educación Ecuador (2013). MIISTERIO DE EDUCACIÓN. Recuperado el 18 de Junio de 2019, de chromeextension://oemmndcbldboiebfnladdacbdfmadadm/https://educacion. gob.ec/wpcontent/uploads/downloads/2016/03/MATE_COMPLETO.pdf

6. Ortiz, A. (2005). www.monografías.com. Recuperado el 30 de 06 de 2019, de https://www.monografias.com/trabajos26/logrosindicadores/logros-indicadores.shtml

7. Torres, R. (2016). La Hora. Recuperado el 12 de Septiembre de 2019, de https://lahora.com.ec/noticia/1101966599/el-examen-ser-bachiller-tendrc3a1-hasta-180-preguntas 